\title{
REFINEMENT OF ADO'S THEOREM IN LOW DIMENSIONS AND APPLICATION IN AFFINE GEOMETRY
}

\author{
YI-FANG KANG AND CHENG-MING BAI*
}

\begin{abstract}
In this paper, we construct a faithful representation with the lowest dimension for every complex Lie algebra in dimension $\leq 4$. In particular, in our construction, in the case that the faithful representation has the same dimension of the Lie algebra, it can induce an étale affine representation with base zero which has a natural and simple form and gives a compatible left-symmetric algebra on the Lie algebra. Such affine representations do not contain any nontrivial one-parameter subgroups of translation.
\end{abstract}

\section{INTRODUCTION}

Let $\mathfrak{g}$ be an $n$-dimensional Lie algebra over a field $\mathbb{F}$ of characteristic zero. A well known theorem due to Ado states that there exists a finite-dimensional faithful representation of $\mathfrak{g}([\mathrm{J}])$. That is, there exists an injective homomorphism $f: \mathfrak{g} \rightarrow \mathfrak{g l}(N)$ of Lie algebras for some $N \in \mathbb{N}$. It is natural to ask what the value of $N$ is in terms of the dimension of $\mathfrak{g}$.

Define an invariant of $\mathfrak{g}$ by $([\mathrm{Bu} 1])$

$$
\mu(\mathfrak{g})=\min \left\{\operatorname{dim}_{\mathbb{F}} \rho \mid \rho \text { is a faithful representation of } \mathfrak{g}\right\} .
$$

By Ado's Theorem, $\mu(\mathfrak{g})$ is finite. It is also known that $\mu(\mathfrak{g}) \leq f(n)$ for a function $f$ only depending on $n$.

In general, except some special cases, it is hard to decide the exact $\mu(\mathfrak{g})$. Even it is not easy to give a suitable estimation on $\mu(\mathfrak{g})$, either, especially in the cases of solvable and nilpotent Lie algebras. Up to now, most of the estimation on $\mu$ for a general nilpotent or solvable Lie algebra is quite "big". For example, for an $n$-dimensional complex solvable Lie algebra $\mathfrak{g}$, there is an estimation $\mu(\mathfrak{g})<1+n+n^{n}([\mathrm{R}])$. And when $\mathfrak{g}$ is an $n$ dimensional nilpotent Lie algebra with nilpotency class $k$ (that is, $\mathfrak{g}^{k} \neq 0, \mathfrak{g}^{k+1}=0$, where $\left.\mathfrak{g}^{i}=\left[\mathfrak{g}, \mathfrak{g}^{i-1}\right], \mathfrak{g}^{1}=\mathfrak{g}\right)$, there is the bound

$$
\mu(\mathfrak{g}) \leq \sum_{j=0}^{k}\left(\begin{array}{l}
n-j \\
k-j
\end{array}\right) p(j)<\frac{3}{\sqrt{n}} 2^{n},
$$

where $\left(\begin{array}{l}n-j \\ k-j\end{array}\right)$ is the binomial number and $p(j)$ is the number of partitions of $j$ ([Bu1-2]).

On the other hand, it is obvious that any $n$-dimensional Lie algebra $\mathfrak{g}$ with trivial center satisfies $\mu(\mathfrak{g}) \leq n$. And any $n$-dimensional real Lie algebra $\mathfrak{g}$ which has a codimension one abelian ideal also satisfies $\mu(\mathfrak{g}) \leq n$ ([GST]). Furthermore, a useful estimation on $\mu$ is given for a Lie algebra $\mathfrak{g}$ with a compatible left-symmetric algebra structure which corresponds to the affine structure on a Lie group $G$ whose Lie algebra is $\mathfrak{g}([\mathrm{M}],[\mathrm{V}])$. For such a $n$-dimensional Lie algebra $\mathfrak{g}$, it is known that $\mu(\mathfrak{g}) \leq n+1$. Left-symmetric algebras are a kind of natural (nonassociative) algebraic systems appearing in many fields in mathematics and mathematical physics, such as affine and symplectic geometry ([K],[DM1-2]),

2000 Mathematics Subject Classification. 17B, 53C.

Key words and phrases. Lie algebra, Ado's Theorem, Left-symmetric algebra, Affine structure.

*Corresponding author. 
integrable systems ([Bo]), classical and quantum Yang-Baxter equation ([ES],[GS]), Poisson brackets and infinite-dimensional Lie algebras $([\mathrm{BN}],[\mathrm{Z}])$, quantum field theory $([\mathrm{CK}])$, operads ([CL]) and so on. Fortunately, there exist left-symmetric algebras on a lot of Lie algebras, in particular, for most of the solvable and nilpotent Lie algebras in low dimensions.

Therefore, it is practicable (and necessary) to decide the exact $\mu$ for certain concrete Lie algebras, at least in low dimensions. In this paper, we find all $\mu(\mathfrak{g})$ for the complex Lie algebras in dimension $\leq 4$. We also give the corresponding faithful representations. The construction is nontrivial since in the case that the faithful representation has the same dimension of the Lie algebra, it can induce an étale affine representation with base zero which has a natural and simple form and gives a compatible left-symmetric algebra structure on the Lie algebra. Although it is based on the example-computation, it can be regarded as a guide for further development. We would like to point out that these results are partly consistent with some results in [GST] $(\mu(\mathfrak{g})$ for the real indecomposable algebras are computed there). The paper is organized as follows. In section 2 , we give some preliminaries and basic results. We omit most of the proofs since they can be found in some related references. In section 3 , we get $\mu(\mathfrak{g})$ for all complex Lie algebras in dimension $\leq 4$ and construct the corresponding faithful representations. In section 4 , we construct the induced étale affine representations and then get the corresponding left-symmetric algebras.

Throughout the paper, all algebras are finite dimensional and over the complex field $\mathbb{C}$.

\section{Preliminaries AND Basic Results}

For some special cases, $\mu(\mathfrak{g})$ can be decided ([Bu1-2]).

Lemma 2.1. Let $\mathfrak{g}$ be an $n$-dimensional commutative Lie algebra. Then $\mu(\mathfrak{g})=[2 \sqrt{n-1}]$, where $[x]$ is the least integer greater or equal than $x$.

Lemma 2.2. Let $\mathfrak{g}$ be a 2-step nilpotent Lie algebra in dimension $n$ with 1-dimensional center. Then $n$ is odd and $\mu(\mathfrak{g})=(n+3) / 2$.

Recall that a filform Lie algebra is an $n$-dimensional nilpotent Lie algebra with nilpotency class $n-1$.

Lemma 2.3. Let $\mathfrak{g}$ be a filform Lie algebra in dimension $n$. If the commutator of $\mathfrak{g}$ is abelian or $n<10$, then $\mu(\mathfrak{g})=n$.

Obviously, there is the (unique) 1-dimensional Lie algebra $\mathbb{C}$. In dimension 2 , there are exactly two (non-isomorphic) Lie algebras: abelian Lie algebra $\mathbb{C}^{2}$ and $\mathfrak{r}_{2}(\mathbb{C})$ with a basis $\left\{e_{1}, e_{2}\right\}$ satisfying $\left[e_{1}, e_{2}\right]=e_{1}$. The classification of 3 and 4 dimensional complex Lie algebras was summarized in [BS] (only the non-zero products are given).

Lemma 2.4. Every complex 3-dimensional Lie algebra is exactly isomorphic to one Lie algebra of the list shown in Table 1.

Lemma 2.5. Every complex 4-dimensional Lie algebra is exactly isomorphic to one Lie algebra of the list shown in Table 2.

On the other hand, we have ([Bai], [BM1-3], [Bu1-4])

Definition 2.6. Let $A$ be a vector space over a field $\mathbb{F}$ with a bilinear product $(x, y) \rightarrow x y$. $A$ is called a left-symmetric algebra if for any $x, y, z \in A$, the associator

$$
(x, y, z)=(x y) z-x(y z)
$$


is symmetric in $x, y$, that is,

$$
(x, y, z)=(y, x, z) \text {, or equivalently }(x y) z-x(y z)=(y x) z-y(x z) .
$$

Proposition 2.7. Let $A$ be a left-symmetric algebra. For any $x, y \in A$, let $L: A \rightarrow \operatorname{gl}(A)$ by $L(x)=L_{x}$, where $L_{x}$ denotes the left multiplication operator, that is, $L_{x}(y)=x y$.

(1) The commutator

$$
[x, y]=x y-y x, \forall x, y \in A,
$$

defines a Lie algebra $\mathfrak{g}(A)$, which is called the sub-adjacent Lie algebra of $A$ and $A$ is called the compatible left-symmetric algebra structure on the Lie algebra $\mathfrak{g}(A)$.

(2) L gives a regular representation of the Lie algebra $\mathfrak{g}(A)$, that is,

$$
\left[L_{x}, L_{y}\right]=L_{[x, y]}, \quad \forall x, y \in A .
$$

Let $\mathfrak{g}$ be a Lie algebra. An affine representation of $\mathfrak{g}$ is a Lie algebra homomorphism $\Phi: \mathfrak{g} \rightarrow \operatorname{aff}(V)$, where $\operatorname{aff}(V)=\left\{\phi=\left(\begin{array}{ll}\varphi & v \\ 0 & 0\end{array}\right) \mid \varphi \in g l(V), v \in V\right\} \subset g l(V \oplus \mathbf{F})$ is the Lie algebra of the group of affine transformations $\operatorname{Aff}(V)=\left\{g=\left(\begin{array}{cc}A & v \\ 0 & 1\end{array}\right) \mid A \in \operatorname{GL}(V), v \in\right.$ $V\} \subset \mathrm{GL}(V \oplus \mathbf{F})$. In addition, $\Phi$ is called an étale affine representation with base point $v$ if there exists $v \in V$ such that the mapping $e v_{v}: \mathfrak{g} \rightarrow V$ defined by $e v_{v}(x)=\Phi(x) v$ for any $x \in \mathfrak{g}$ is an isomorphism. It is known that $\Phi=(\rho, q): \mathfrak{g} \rightarrow \operatorname{aff}(V)$ with $\Phi(x)=\rho(x)+q(x)$ is an affine representation if and only if $\rho: \mathfrak{g} \rightarrow g l(V)$ is a representation of $\mathfrak{g}$ and $q: \mathfrak{g} \rightarrow V$ is a linear map satisfying

$$
q[x, y]=\rho(x) q(y)-\rho(y) q(x), \forall x, y \in \mathfrak{g} .
$$

Proposition 2.8. Let $\mathfrak{g}$ be a Lie algebra. Then there is a compatible left-symmetric algebra structure on $\mathfrak{g}$ if and only if $\mathfrak{g}$ has an étale affine representation.

In fact, let $\Phi=(\rho, q): \mathfrak{g} \rightarrow \operatorname{aff}(V)$ be an étale affine representation of $\mathfrak{g}$, then

$$
x * y=e v_{v}^{-1}\left[\rho(x) e v_{v}(y)\right], \quad \forall x, y \in \mathfrak{g},
$$

defines a left-symmetric algebra structure on $\mathfrak{g}$. Conversely, for a left-symmetric algebra $A, \Phi=(L, i d)$ is an étale affine representation of $\mathfrak{g}(A)$ with base 0 , where $i d$ is the identity transformation on $\mathfrak{g}(A)$. Moreover, if $A$ is a left-symmetric algebra, then $N(A)=\{x \in$ $\left.A \mid L_{x}=0\right\}$ is an ideal of $A$ which is called a kernel ideal.

Proposition 2.9. ([M]) A left-symmetric algebra A has a zero kernel ideal if and only if its corresponding étale affine representation does not contain any nontrivial one-parameter subgroups of translation.

TABLE 1.

\begin{tabular}{|c|c|c|}
\hline $\mathfrak{g}$ & $\mu(\mathfrak{g})$ & Lie brackets \\
\hline $\mathbb{C}^{3}$ & 3 & - \\
\hline $\mathfrak{n}_{3}(\mathbb{C})$ & 3 & {$\left[e_{1}, e_{2}\right]=e_{3}$} \\
\hline $\mathfrak{r}_{2}(\mathbb{C}) \oplus \mathbb{C}$ & 2 & {$\left[e_{1}, e_{2}\right]=e_{1}$} \\
\hline $\mathfrak{r}_{3}(\mathbb{C})$ & 3 & {$\left[e_{1}, e_{2}\right]=e_{2},\left[e_{1}, e_{3}\right]=e_{2}+e_{3}$} \\
\hline $\mathfrak{r}_{3, \lambda}(\mathbb{C})$ & 3 & {$\left[e_{1}, e_{2}\right]=e_{2},\left[e_{1}, e_{3}\right]=\lambda e_{3}, \lambda \in \mathbb{C}^{*},|\lambda|<1$ or $\lambda=e^{i \theta}, 0 \leq \theta \leq \pi$} \\
\hline $\mathfrak{s l}_{2}(\mathbb{C})$ & 2 & {$\left[e_{1}, e_{2}\right]=e_{3},\left[e_{1}, e_{3}\right]=-2 e_{2},\left[e_{2}, e_{3}\right]=2 e_{2}$} \\
\hline
\end{tabular}


TABLE 2.

\begin{tabular}{|c|c|c|}
\hline$\overline{\mathfrak{g}}$ & $\mu(\mathfrak{g})$ & Lie brackets \\
\hline $\mathbb{C}^{4}$ & 4 & - \\
\hline $\mathfrak{n}_{3}(\mathbb{C}) \oplus \mathbb{C}$ & 3 & {$\left[e_{1}, e_{2}\right]=e_{3}$} \\
\hline $\mathfrak{r}_{2}(\mathbb{C}) \oplus \mathbb{C}^{2}$ & 3 & {$\left[e_{1}, e_{2}\right]=e_{1}$} \\
\hline $\mathfrak{r}_{3}(\mathbb{C}) \oplus \mathbb{C}$ & 3 & {$\left[e_{1}, e_{2}\right]=e_{2},\left[e_{1}, e_{3}\right]=e_{2}+e_{3}$} \\
\hline $\mathfrak{r}_{3, \lambda}(\mathbb{C}) \oplus \mathbb{C}$ & 3 & {$\left[e_{1}, e_{2}\right]=e_{2},\left[e_{1}, e_{3}\right]=\lambda e_{3}, \lambda \in \mathbb{C}^{*},|\lambda|<1$ or $\lambda=e^{i \theta}, 0 \leq \theta \leq \pi$} \\
\hline $\mathfrak{r}_{2}(\mathbb{C}) \oplus \mathfrak{r}_{2}(\mathbb{C})$ & 3 & {$\left[e_{1}, e_{2}\right]=e_{1},\left[e_{3}, e_{4}\right]=e_{3}$} \\
\hline $\mathfrak{s l}_{2}(\mathbb{C}) \oplus \mathbb{C}$ & 2 & {$\left[e_{1}, e_{2}\right]=e_{3},\left[e_{1}, e_{3}\right]=-2 e_{1},\left[e_{2}, e_{3}\right]=2 e_{2}$} \\
\hline $\mathfrak{n}_{4}(\mathbb{C})$ & 4 & {$\left[e_{1}, e_{2}\right]=e_{3},\left[e_{1}, e_{3}\right]=e_{4}$} \\
\hline $\mathfrak{g}_{1}(\alpha)$ & 4 & {$\left[e_{1}, e_{2}\right]=e_{2},\left[e_{1}, e_{3}\right]=e_{3},\left[e_{1}, e_{4}\right]=\alpha e_{4}, \alpha \in \mathbb{C}^{*}$} \\
\hline $\mathfrak{g}_{2}(\alpha, \beta)$ & 4 & $\begin{array}{c}{\left[e_{1}, e_{2}\right]=e_{3},\left[e_{1}, e_{3}\right]=e_{4},\left[e_{1}, e_{4}\right]=\alpha e_{2}-\beta e_{3}+e_{4}} \\
\alpha \in \mathbb{C}^{*}, \beta \in \mathbb{C} \text { or } \alpha, \beta=0\end{array}$ \\
\hline $\mathfrak{g}_{3}(\alpha)$ & 4 & {$\left[e_{1}, e_{2}\right]=e_{3},\left[e_{1}, e_{3}\right]=e_{4},\left[e_{1}, e_{4}\right]=\alpha\left(e_{2}+e_{3}\right), \alpha \in \mathbb{C}^{*}$} \\
\hline $\mathfrak{g}_{4}$ & 4 & {$\left[e_{1}, e_{2}\right]=e_{3},\left[e_{1}, e_{3}\right]=e_{4},\left[e_{1}, e_{4}\right]=e_{2}$} \\
\hline $\mathfrak{g}_{5}$ & 4 & {$\left[e_{1}, e_{2}\right]=\frac{1}{3} e_{2}+e_{3},\left[e_{1}, e_{3}\right]=\frac{1}{3} e_{3},\left[e_{1}, e_{4}\right]=\frac{1}{3} e_{4}$} \\
\hline $\mathfrak{g}_{6}$ & 3 & {$\left[e_{1}, e_{2}\right]=e_{2},\left[e_{1}, e_{3}\right]=e_{3},\left[e_{1}, e_{4}\right]=2 e_{4},\left[e_{2}, e_{3}\right]=e_{4}$} \\
\hline $\mathfrak{g}_{7}$ & 3 & {$\left[e_{1}, e_{2}\right]=e_{3},\left[e_{1}, e_{3}\right]=e_{2},\left[e_{2}, e_{3}\right]=e_{4}$} \\
\hline $\mathfrak{g}_{8}(\alpha)$ & $\begin{array}{l}3\left(\alpha \neq \frac{1}{4}\right) \\
4\left(\alpha=\frac{1}{4}\right)\end{array}$ & {$\left[e_{1}, e_{2}\right]=e_{3},\left[e_{1}, e_{3}\right]=-\alpha e_{2}+e_{3},\left[e_{1}, e_{4}\right]=e_{4},\left[e_{2}, e_{3}\right]=e_{4}, \alpha \in \mathbb{C}$} \\
\hline
\end{tabular}

Theorem 2.10. Let $\mathfrak{g}$ be a Lie algebra. If there exists an étale affine representation $\Phi=(\rho, q)$ of $\mathfrak{g}$ such that $\rho$ is a faithful representation of $\mathfrak{g}$, then the corresponding leftsymmetric algebra has the zero kernel ideal. Therefore $\Phi$ does not contain any nontrivial one-parameter subgroups of translation.

Proof Let $x \in N(\mathfrak{g})$. By equation (2.6), we have $e v_{v}^{-1}\left[\rho(x) e v_{v}(y)\right]=0$ for every $y \in \mathfrak{g}$. Since $e v_{v}$ is a linear isomorphism, $\rho(x) z=0$ for every $z \in \mathfrak{g}$. Then $x \in \operatorname{Ker} \rho$. Since $\rho$ is faithful, $x=0$.

\section{The LOWESt Dimensional faithful RePRESEntations of LiE ALgEBras in DIMENSION $\leq 4$}

Obviously, $\mu(\mathfrak{g})=1$ if only if $\mathfrak{g}$ is isomorphic to $\mathbb{C}$. Furthermore, by Lemma 2.1 , we have

Corollary 3.1. With the notations given in section 2,

$$
\mu(\mathbb{C})=1, \mu\left(\mathbb{C}^{2}\right)=2, \mu\left(\mathbb{C}^{3}\right)=3, \mu\left(\mathbb{C}^{4}\right)=4 .
$$

By Lemma 2.2, we have

Corollary 3.2. With the notations given in section $2, \mu\left(\mathfrak{n}_{3}(\mathbb{C})\right)=3$.

By Lemma 2.3, we have

Corollary 3.3. With the notations given in section $2, \mu\left(\mathfrak{n}_{4}(\mathbb{C})\right)=4$.

Lemma 3.4. Let $\mathfrak{g}$ be a Lie algebra in dimension $n \leq 4$. If $\mathfrak{g}$ is 2-solvable (that is, $[\mathfrak{g}, \mathfrak{g}]$ is a commutative Lie algebra) and $\operatorname{dim}[\mathfrak{g}, \mathfrak{g}]=n-1$, then $\mu(\mathfrak{g}) \geq n$.

Proof We assume that $\mathfrak{g}$ has a faithful representation $(\rho, V)$ in dimension $m<n$. Since $\mathfrak{g}$ is solvable, by Lie's theorem, there exists a basis $\left\{e_{1}, \cdots, e_{m}\right\}$ of $V$ such that $\rho(x) \in \mathfrak{g l}(m, \mathbb{C})$ is upper-triangle for all $x \in \mathfrak{g}$. Therefore, $\rho([\mathfrak{g}, \mathfrak{g}])$ is in the space of strictly upper-triangle matrices whose dimension is $m(m-1) / 2$. When $1<n \leq 3$, we 
have $m(m-1) / 2<n-1$, which is a contradiction. When $n=4$, we can suppose $m=3$ since $\operatorname{dim}[\mathfrak{g}, \mathfrak{g}]=3$. Then $\rho([\mathfrak{g}, \mathfrak{g})]$ is exactly the Lie algebra of the $3 \times 3$ strictly uppertriangle matrices which is not commutative. It is contradictive to the fact that $[\mathfrak{g}, \mathfrak{g}]$ is commutative. Therefore, the conclusion holds.

Corollary 3.5. With the notations given in section 2,

$$
\begin{aligned}
& (n=2) \mu\left(\mathfrak{r}_{2}(\mathbb{C})\right)=2 ; \\
& (n=3) \mu\left(\mathfrak{r}_{3}(\mathbb{C})\right)=\mu\left(\mathfrak{r}_{3, \lambda}(\mathbb{C})\right)=3 ; \\
& (n=4) \mu\left(\mathfrak{g}_{1}(\alpha)\right)=\mu\left(\mathfrak{g}_{2}(\alpha, \beta)\left(\alpha \in \mathbb{C}^{*}, \beta \in \mathbb{C}\right)\right)=\mu\left(\mathfrak{g}_{3}(\alpha)\right)=\mu\left(\mathfrak{g}_{4}\right)=\mu\left(\mathfrak{g}_{5}\right)=4 .
\end{aligned}
$$

Proof Let $\mathfrak{g}$ be one of the above Lie algebras. Then $\mathfrak{g}$ satisfies the conditions in Lemma 3.4 and its adjoint representation is faithful since $\mathfrak{g}$ is centerless. Therefore $\mu(\mathfrak{g})=$ $n$.

Lemma 3.6. Let $\mathfrak{g}$ be a complex centerless Lie algebra. Then $\mu(\mathfrak{g})=\mu(\mathfrak{g} \oplus \mathbb{C})$.

Proof Let $\mu(\mathfrak{g})=n$ and $\rho: \mathfrak{g} \rightarrow \mathfrak{g l}(n, \mathbb{C})$ be a faithful representation. Obviously, $\mu(\mathfrak{g} \oplus \mathbb{C}) \geq n$. Since the identity matrix $I_{n} \notin \rho(\mathfrak{g}), \rho(\mathfrak{g}) \oplus \mathbb{C} \cdot I_{n}$ gives a faithful representation of $\mathfrak{g} \oplus \mathbb{C}$. Therefore $\mu(\mathfrak{g} \oplus \mathbb{C})=\mu(\mathfrak{g})=n$.

Corollary 3.7. With the notations given in section 2,

$$
\mu\left(\mathfrak{r}_{2}(\mathbb{C}) \oplus \mathbb{C}\right)=2, \mu\left(\mathfrak{r}_{3}(\mathbb{C}) \oplus \mathbb{C}\right)=\mu\left(\mathfrak{r}_{3, \lambda}(\mathbb{C}) \oplus \mathbb{C}\right)=3 .
$$

Theorem 3.8. Let $\mathfrak{g}$ be a complex Lie algebra. If $\mu(\mathfrak{g})=2$, then $\mathfrak{g}$ is isomorphic to one of the following algebras:

$$
\mathbb{C}^{2}, \mathfrak{r}_{2}(\mathbb{C}), \mathfrak{r}_{2}(\mathbb{C}) \oplus \mathbb{C}, \mathfrak{s l}_{2}(\mathbb{C}), \mathfrak{s l}_{2}(\mathbb{C}) \oplus \mathbb{C} .
$$

Proof If $\mu(\mathfrak{g})=2$, then there exists an injective homomorphism $f: \mathfrak{g} \rightarrow \mathfrak{g l}(2, \mathbb{C})=$ $\mathfrak{s l}_{2}(\mathbb{C}) \oplus \mathbb{C}$ of Lie algebras. Hence $\operatorname{dim} \mathfrak{g} \leq 4$ and $\operatorname{dim} \mathfrak{g}=4$ if and only if $\mathfrak{g}$ is isomorphic to $\mathfrak{g l}(2, \mathbb{C}) . \quad \mu(\mathfrak{s l}(2, \mathbb{C}))=2$ since the fundamental representation of $\mathfrak{s l}(2, \mathbb{C})$ is faithful. Therefore we have found all $\mu(\mathfrak{g})$ when $\mathfrak{g}$ is a complex Lie algebra in dimension 2 or 3 . Hence the conclusion holds.

There are still the following 4-dimensional Lie algebras whose $\mu(\mathfrak{g}) \mathrm{s}$ have not been decided:

$$
\mathfrak{n}_{3}(\mathbb{C}) \oplus \mathbb{C}, \mathfrak{r}_{2}(\mathbb{C}) \oplus \mathbb{C}^{2}, \mathfrak{r}_{2}(\mathbb{C}) \oplus \mathfrak{r}_{2}(\mathbb{C}), \mathfrak{g}_{2}(0,0), \mathfrak{g}_{6}, \mathfrak{g}_{7}, \mathfrak{g}_{8}(\alpha) .
$$

Let $\mathfrak{g}$ be one of the above Lie algebras. Then $\mathfrak{g}$ is solvable and $\mu(\mathfrak{g}) \geq 3$. It is known that there exists a left-symmetric algebra structure on $\mathfrak{g}([\mathrm{Bu} 1])$. So $\mu(\mathfrak{g}) \leq 5$ (in fact, $\mu(\mathfrak{g}) \leq 4$ from [GST] or the following discussion). If we can construct a 3-dimensional faithful representation, then $\mu(\mathfrak{g})=3$. Otherwise, we need to show that $\mu(\mathfrak{g})>3$ and if we can construct a 4 -dimensional faithful representation, then $\mu(\mathfrak{g})=4$.

Proposition 3.9. With the notations given in section 2 , if $\mathfrak{g}$ is isomorphic to one of the following algebras: $\mathfrak{n}_{3}(\mathbb{C}) \oplus \mathbb{C}, \mathfrak{r}_{2}(\mathbb{C}) \oplus \mathbb{C}^{2}, \mathfrak{r}_{2}(\mathbb{C}) \oplus \mathfrak{r}_{2}(\mathbb{C}), \mathfrak{g}_{6}, \mathfrak{g}_{7}, \mathfrak{g}_{8}(\alpha)\left(\alpha \neq \frac{1}{4}\right)$, then $\mu(\mathfrak{g})=3$.

Proof We can construct a 3-dimensional faithful representation for every above Lie algebra. The construction is given in Table 3 .

Proposition 3.10. With the notations given in section 2, if $\mathfrak{g}$ is isomorphic to $\mathfrak{g}_{2}(0,0)$ or $\mathfrak{g}_{8}\left(\frac{1}{4}\right)$, then $\mu(\mathfrak{g})=4$.

Proof Let $\mathfrak{g}$ be $\mathfrak{g}_{2}(0,0)$ or $\mathfrak{g}_{8}\left(\frac{1}{4}\right)$ and $\left\{e_{1}, e_{2}, e_{3}, e_{4}\right\}$ be a basis of $\mathfrak{g}$ with the Lie brackets in Table 2. Suppose that $(\rho, V)$ is a 3-dimensional faithful representation of $\mathfrak{g}$. By Lie's 
theorem, we can select a basis of $V$ such that $\rho(x) \in \mathfrak{g l}(3, \mathbb{C})$ is upper-triangle for all $x \in \mathfrak{g}$. That is, we can write $\rho\left(e_{i}\right)(i=1,2,3,4)$ as follow:

$\left(\begin{array}{ccc}a_{11} & a_{12} & a_{13} \\ 0 & a_{22} & a_{23} \\ 0 & 0 & a_{33}\end{array}\right),\left(\begin{array}{ccc}b_{11} & b_{12} & b_{13} \\ 0 & b_{22} & b_{23} \\ 0 & 0 & b_{33}\end{array}\right),\left(\begin{array}{ccc}c_{11} & c_{12} & c_{13} \\ 0 & c_{22} & c_{23} \\ 0 & 0 & c_{33}\end{array}\right),\left(\begin{array}{ccc}d_{11} & d_{12} & d_{13} \\ 0 & d_{22} & d_{23} \\ 0 & 0 & d_{33}\end{array}\right)$.

(1) $\mathfrak{g}=\mathfrak{g}_{2}(0,0)$. Then by the Lie brackets, we have

(a) $\quad c_{11}=c_{22}=c_{33}=d_{11}=d_{22}=d_{33}=0$

(b) $\quad c_{12}=b_{12}\left(a_{11}-a_{22}\right)+a_{12}\left(b_{22}-b_{11}\right), c_{23}=b_{23}\left(a_{22}-a_{33}\right)+a_{23}\left(b_{33}-b_{22}\right)$;

(c) $\quad c_{13}=b_{13}\left(a_{11}-a_{33}\right)+a_{12} b_{23}+a_{13} b_{33}-b_{12} a_{23}-b_{11} a_{13}$;

(d) $\quad d_{12}=c_{12}\left(a_{11}-a_{22}\right), d_{23}=c_{23}\left(a_{22}-a_{33}\right)$;

(e) $\quad d_{13}=c_{13}\left(a_{11}-a_{33}\right)+a_{12} c_{23}-a_{23} c_{12}$;

(f) $\quad c_{12}\left(b_{11}-b_{22}\right)=0, \quad d_{12}\left(b_{11}-b_{22}\right)=0$

(g) $\quad c_{23}\left(b_{22}-b_{33}\right)=0, \quad d_{23}\left(b_{22}-b_{33}\right)=0$

(h) $\quad c_{13}\left(b_{11}-b_{33}\right)+b_{12} c_{23}-b_{23} c_{12}=0$;

(i) $\quad d_{13}\left(b_{11}-b_{33}\right)+b_{12} d_{23}-b_{23} d_{12}=0$;

(j) $\quad c_{12}\left(a_{11}-a_{22}\right)^{2}=c_{12}\left(a_{11}-a_{22}\right), c_{23}\left(a_{22}-a_{33}\right)^{2}=c_{23}\left(a_{22}-a_{33}\right)$.

Case (1-i): $b_{11}, b_{22}$ and $b_{33}$ do not equal to each other. Then $b_{11} \neq b_{22}$ or $b_{22} \neq b_{33}$. Without losing the generality, we assume $b_{22} \neq b_{33}$ (in fact, the discussion for the case $b_{11} \neq b_{22}$ is similar). Therefore $c_{23}=d_{23}=0$ by $(g)$. Moreover, $c_{12}$ and $d_{12}$ cannot be zero at the same time. Otherwise, $\rho\left(e_{3}\right)$ is proportional to $\rho\left(e_{4}\right)$ by $(a)$, which is a contradiction. Hence we have $b_{11}=b_{22}$ by $(f)$ and $c_{12} \neq 0$ by $(d)$. Thus, using $(b)$, we know $c_{12}=b_{12}\left(a_{11}-a_{22}\right) \neq 0$ which gives $b_{12} \neq 0$ and $a_{11} \neq a_{22}$. By $(j)$, we have $a_{11}-a_{22}=1$. On the other hand, by $(h)$ and $c_{23}=0$, we know that $c_{13}\left(b_{11}-b_{33}\right)=c_{12} b_{23}$. If $c_{13}=0$, then $b_{23}=0$ since $c_{12} \neq 0$. Therefore, by $(i)$ and $d_{23}=0$, we have $d_{13}\left(b_{11}-b_{33}\right)=0$. So $d_{13}=0$. It gives that $\rho\left(e_{3}\right)$ is proportional to $\rho\left(e_{4}\right)$ by $(a)$, which is a contradiction. If $c_{13} \neq 0$, then $b_{23} \neq 0$. By $(d)$ and $a_{11}-a_{22}=1$, we have $c_{12}=d_{12}$. By $(i)$ and $d_{23}=0$, we have $d_{13}\left(b_{11}-b_{33}\right)=d_{12} b_{23}=c_{12} b_{23}=c_{13}\left(b_{11}-b_{33}\right)$. So $d_{13}=c_{13}$. Therefore $\rho\left(e_{3}\right)=\rho\left(e_{4}\right)$, which is a contradiction.

Case (1-ii): $b_{11}=b_{22}=b_{33}$. Set $e=\rho\left(e_{2}\right)-b_{11} I_{3} \neq 0$ which is a strictly upper-triangle matrix. Since $\left[e, \rho\left(e_{3}\right)\right]=\left[e, \rho\left(e_{4}\right)\right]=\left[\rho\left(e_{3}\right), \rho\left(e_{4}\right)\right]=0$, we have that $\left\{e, \rho\left(e_{3}\right), \rho\left(e_{4}\right)\right\}$ spans an abelian subalgebra of the Lie algebra of the $3 \times 3$ strictly upper-triangle matrices. Therefore $e$ is in the vector space spanned by $\rho\left(e_{3}\right)$ and $\rho\left(e_{4}\right)$. We assume $e=\alpha \rho\left(e_{3}\right)+$ $\beta \rho\left(e_{4}\right)$. So $\rho\left(e_{3}\right)=\rho\left(\left[e_{1}, e_{2}\right]\right)=\left[\rho\left(e_{1}\right), \rho\left(e_{2}\right)\right]=\left[\rho\left(e_{1}\right), e\right]=(\alpha+\beta) \rho\left(e_{4}\right)$, which is again a contradiction.

(2) $\mathfrak{g}=\mathfrak{g}_{8}\left(\frac{1}{4}\right)$. With a similar discussion as in the above case (1), we can know that $\mu\left(\mathfrak{g}_{8}\left(\frac{1}{4}\right)\right)>3$.

On the other hand, for $\mathfrak{g}$ being $\mathfrak{g}_{2}(0,0)$ or $\mathfrak{g}_{8}\left(\frac{1}{4}\right)$, we can construct a 4-dimensional faithful representation given in Table 3.

At the end of this section, for every complex Lie algebra in dimension $\leq 4$, we construct a faithful representations with the lowest dimension and list it in the third column in Table 3. Let $\left\{e_{1}, \cdots, e_{m}\right\}$ be a basis of $\mathfrak{g}$ and $e_{i j}$ be the standard basis of the general linear Lie algebra $\mathfrak{g l}(n, \mathbb{C})$. We also give the corresponding left-symmetric algebras in the fourth column in Table 3 which will be discussed in next section. 


\section{TABLE 3}

\begin{tabular}{|c|c|c|c|}
\hline $\mathfrak{g}$ & $\mu(\mathfrak{g})$ & Faithful representation & Left-symmetric algebra \\
\hline $\mathbb{C}$ & 1 & $e_{1} \mapsto e_{11}$ & $e_{1} * e_{1}=e_{1}$ \\
\hline $\mathbb{C}^{2}$ & 2 & $e_{1} \mapsto e_{11}, e_{2} \mapsto e_{22}$ & $e_{1} * e_{1}=e_{1}, e_{2} * e_{2}=e_{2}$ \\
\hline $\mathfrak{r}_{2}(\mathbb{C})$ & 2 & $e_{1} \mapsto e_{12}, e_{2} \mapsto e_{22}$ & $e_{1} * e_{2}=e_{1}, e_{2} * e_{2}=e_{2}$ \\
\hline $\mathbb{C}^{3}$ & 3 & $e_{1} \mapsto e_{11}, e_{2} \mapsto e_{22}, e_{3} \mapsto e_{33}$ & $e_{1} * e_{1}=e_{1}, e_{2} * e_{2}=e_{2}, e_{3} * e_{3}=e_{3}$ \\
\hline $\mathfrak{n}_{3}(\mathbb{C})$ & 3 & $\begin{array}{c}e_{1} \mapsto I_{3}+e_{12}-e_{23}, \\
e_{2} \mapsto e_{12}+e_{23}, e_{3} \mapsto 2 e_{13}\end{array}$ & $\begin{array}{c}e_{1} * e_{1}=e_{1}-e_{2}+\frac{1}{2} e_{3}, \\
e_{1} * e_{2}=e_{2}+\frac{1}{2} e_{3}, e_{1} * e_{3}=e_{3}, \\
e_{2} * e_{1}=e_{2}-\frac{1}{2} e_{3}, e_{2} * e_{2}=\frac{1}{2} e_{3}, \\
e_{3} * e_{1}=e_{3}\end{array}$ \\
\hline $\mathfrak{r}_{2}(\mathbb{C}) \oplus \mathbb{C}$ & 2 & $e_{1} \mapsto e_{12}, e_{2} \mapsto e_{22}, e_{3} \mapsto I_{2}$ & \\
\hline $\mathfrak{r}_{3}(\mathbb{C})$ & 3 & $\begin{array}{c}e_{1} \mapsto e_{12}-e_{33} \\
e_{2} \mapsto e_{13}, e_{3} \mapsto e_{23}\end{array}$ & $\begin{array}{c}e_{1} * e_{1}=-e_{1}+e_{2}, e_{1} * e_{3}=e_{2} \\
e_{2} * e_{1}=-e_{2}, e_{3} * e_{1}=-e_{3}\end{array}$ \\
\hline$\overline{\mathfrak{r}_{3, \lambda}(\mathbb{C})}$ & 3 & $\begin{array}{c}e_{1} \mapsto-e_{11}+(\lambda-1) e_{33}, \\
e_{2} \mapsto e_{21}, e_{3} \mapsto e_{31}\end{array}$ & $\begin{array}{c}e_{1} * e_{1}=-e_{1}+\left(\lambda^{2}-\lambda\right) e_{3} \\
e_{1} * e_{3}=(\lambda-1) e_{3}, e_{2} * e_{1}=-e_{2} \\
e_{3} * e_{1}=-e_{3}\end{array}$ \\
\hline $\mathfrak{s l}_{2}(\mathbb{C})$ & 2 & $\begin{array}{c}e_{1} \mapsto e_{12}, e_{2} \mapsto e_{21}, \\
e_{3} \mapsto e_{11}-e_{22}\end{array}$ & \\
\hline $\mathbb{C}^{4}$ & 4 & $\begin{aligned} & e_{1} \mapsto e_{11}, e_{2} \mapsto e_{22}, \\
& e_{3} \mapsto e_{33}, e_{4} \mapsto e_{44}\end{aligned}$ & $\begin{array}{l}e_{1} * e_{1}=e_{1}, e_{2} * e_{2}=e_{2} \\
e_{3} * e_{3}=e_{3}, e_{4} * e_{4}=e_{4}\end{array}$ \\
\hline $\mathfrak{n}_{3}(\mathbb{C}) \oplus \mathbb{C}$ & 3 & $\begin{array}{c}e_{1} \mapsto e_{12}, e_{2} \mapsto e_{23}, \\
e_{3} \mapsto e_{13}, e_{4} \mapsto I_{3}\end{array}$ & \\
\hline $\mathfrak{r}_{2}(\mathbb{C}) \oplus \mathbb{C}^{2}$ & 3 & $\begin{array}{c}e_{1} \mapsto e_{13}, e_{2} \mapsto e_{33}, \\
e_{3} \mapsto e_{22}, e_{4} \mapsto I_{3}\end{array}$ & \\
\hline $\mathfrak{r}_{3}(\mathbb{C}) \oplus \mathbb{C}$ & 3 & $\begin{array}{c}e_{1} \mapsto e_{12}-e_{33}, e_{2} \mapsto e_{13}, \\
e_{3} \mapsto e_{23}, e_{4} \mapsto I_{3} \\
\end{array}$ & \\
\hline $\mathfrak{r}_{3, \lambda}(\mathbb{C}) \oplus \mathbb{C}$ & 3 & $\begin{array}{c}e_{1} \mapsto e_{11}+(1-\lambda) e_{33}, \\
e_{2} \mapsto e_{12}, e_{3} \mapsto e_{13}, e_{4} \mapsto I_{3}\end{array}$ & \\
\hline $\mathfrak{r}_{2}(\mathbb{C}) \oplus \mathfrak{r}_{2}(\mathbb{C})$ & 3 & $\begin{aligned} e_{1} & \mapsto e_{13}, e_{2} \mapsto e_{33}, \\
e_{3} & \mapsto e_{12}, e_{4} \mapsto e_{22}\end{aligned}$ & \\
\hline $\mathfrak{s l}_{2}(\mathbb{C}) \oplus \mathbb{C}$ & 2 & $\begin{array}{c}e_{1} \mapsto e_{12}, e_{2} \mapsto e_{21}, \\
e_{3} \mapsto e_{11}-e_{22}, e_{4} \mapsto I_{2}\end{array}$ & \\
\hline $\mathfrak{n}_{4}(\mathbb{C})$ & 4 & $\begin{array}{c}e_{1} \mapsto I_{4}+e_{12}+e_{23} \\
e_{2} \mapsto e_{34}, e_{3} \mapsto e_{24}, e_{4} \mapsto e_{14}\end{array}$ & $\begin{array}{c}e_{1} * e_{1}=e_{1}+e_{3}+2 e_{4} \\
e_{1} * e_{2}=e_{2}+e_{3}, e_{1} * e_{3}=e_{3}+e_{4} \\
e_{1} * e_{4}=e_{4}, e_{2} * e_{1}=e_{2} \\
e_{3} * e_{1}=e_{3}, e_{4} * e_{1}=e_{4}\end{array}$ \\
\hline$\overline{\mathfrak{g}_{1}(\alpha)}$ & 4 & $\begin{array}{c}e_{1} \mapsto-e_{11}+(\alpha-1) e_{44} \\
e_{2} \mapsto e_{21}, e_{3} \mapsto e_{31}, e_{4} \mapsto e_{41}\end{array}$ & $\begin{array}{c}e_{1} * e_{1}=-e_{1}+\left(\alpha^{2}-\alpha\right) e_{4} \\
e_{1} * e_{4}=(\alpha-1) e_{4}, e_{2} * e_{1}=-e_{2} \\
e_{3} * e_{1}=-e_{3}, e_{4} * e_{1}=-e_{4}\end{array}$ \\
\hline $\mathfrak{g}_{2}(\alpha, \beta)$ & 4 & $\begin{array}{c}e_{1} \mapsto-b e_{11}-x e_{33}-y e_{44}+e_{32} \\
+e_{43}, e_{2} \mapsto e_{21}, e_{3} \mapsto b e_{21}+e_{31} \\
e_{4} \mapsto b^{2} e_{21}+(2 b-x) e_{31}+e_{41}, \\
\text { where } b \neq 0 \text { satisfies } \\
b^{3}=\alpha-\beta b+b^{2}, \\
x^{2}-3 b x+3 b^{2}=-\beta+2 b-x, \\
y=3 b-1-x\end{array}$ & $\begin{array}{c}e_{1} * e_{1}=-b e_{1}+\left(2 b^{3} y-b^{2} y-2 b^{3}+\right. \\
\left.b^{2}+b x y-b x y^{2}\right) e_{2}+\left(4 b^{2}-3 b+\right. \\
\left.2 b^{2} y-b x y-x y+x y^{2}-2 b y^{2}\right) e_{3} \\
+\left(2-2 b-b y+y^{2}\right) e_{4} \\
e_{1} * e_{2}=e_{3}-b e_{2}, e_{1} * e_{3}=e_{4}-b e_{3}, \\
e_{1} * e_{4}=\alpha e_{2}-\beta e_{3}+(1-b) e_{4}, \\
e_{2} * e_{1}=-b e_{2}, e_{3} * e_{1}=-b e_{3}, \\
e_{4} * e_{1}=-b e_{4}\end{array}$ \\
\hline
\end{tabular}


TABLE 3 (Continued)

\begin{tabular}{|c|c|c|c|}
\hline $\mathfrak{g}$ & $\mu(\mathfrak{g})$ & Faithful representation & Left-symmetric algebra \\
\hline $\mathfrak{g}_{3}(\alpha)$ & 4 & $\begin{array}{c}e_{1} \mapsto-b e_{11}-x e_{33}-y e_{44}+e_{32} \\
+e_{43}, e_{2} \mapsto e_{21}, e_{3} \mapsto b e_{21}+e_{31}, \\
e_{4} \mapsto b^{2} e_{21}+(2 b-x) e_{31}+e_{41}, \\
\text { where } b^{3}=\alpha(b+1), \\
x^{2}-3 b x+3 b^{2}-\alpha=0 \\
y=3 b-x\end{array}$ & $\begin{array}{c}e_{1} * e_{1}=-b e_{1}+\left(2 b^{3} y-2 b^{3}+b x y-\right. \\
\left.b x y^{2}\right) e_{2}+\left(4 b^{2}-b-4 b^{2} y+b x y-\right. \\
\left.x y+x y^{2}\right) e_{3}+\left(1-2 b-b y+y^{2}\right) e_{4}, \\
e_{1} * e_{2}=e_{3}-b e_{2}, e_{1} * e_{3}=e_{4}-b e_{3} \\
e_{1} * e_{4}=-b e_{4}+\alpha\left(e_{2}+e_{3}\right) \\
e_{2} * e_{1}=-b e_{2}, e_{3} * e_{1}=-b e_{3}, \\
e_{4} * e_{1}=-b e_{4}\end{array}$ \\
\hline $\mathfrak{g}_{4}$ & 4 & $\begin{aligned} & e_{1} \mapsto-e_{11}+e_{32}-\frac{3+\sqrt{3} i}{2} e_{33} \\
&+e_{43}-\frac{3-\sqrt{3} i}{2} e_{44} \\
& e_{2} \mapsto e_{21}, e_{3} \mapsto e_{21}+e_{31}, \\
& e_{4} \mapsto e_{21}+\frac{1-\sqrt{3} i}{2} e_{31}+e_{41}\end{aligned}$ & $\begin{array}{c}e_{1} * e_{1}=-e_{1}+\frac{-1+\sqrt{3} i}{2} e_{2}+\frac{3+\sqrt{3} i}{2} e_{3} \\
-(1+\sqrt{3} i) e_{4}, e_{1} * e_{2}=e_{3}-e_{2}, \\
e_{1} * e_{3}=e_{4}-e_{3}, e_{1} * e_{4}=e_{2}-e_{4}, \\
e_{2} * e_{1}=-e_{2}, e_{3} * e_{1}=-e_{3}, \\
e_{4} * e_{1}=-e_{4}\end{array}$ \\
\hline $\mathfrak{g}_{5}$ & 4 & $\begin{array}{c}e_{1} \mapsto-\frac{1}{3} e_{44}+e_{23}, \\
e_{2} \mapsto e_{34}, e_{3} \mapsto e_{24}, e_{4} \mapsto e_{14}\end{array}$ & $\begin{array}{c}e_{1} * e_{1}=-\frac{1}{3} e_{1}+\frac{1}{3} e_{3} \\
e_{1} * e_{2}=e_{3}, e_{2} * e_{1}=-\frac{1}{3} e_{2} \\
e_{3} * e_{1}=-\frac{1}{3} e_{3}, e_{4} * e_{1}=-\frac{1}{3} e_{4}\end{array}$ \\
\hline $\mathfrak{g}_{6}$ & 3 & $\begin{array}{c}e_{1} \mapsto e_{11}-e_{33}, e_{2} \mapsto \frac{\sqrt{2}}{2}\left(e_{12}-e_{23}\right), \\
e_{3} \mapsto \frac{\sqrt{2}}{2}\left(e_{12}+e_{23}\right), e_{4} \mapsto e_{13}\end{array}$ & \\
\hline $\mathfrak{g}_{7}$ & 3 & $\begin{array}{c}e_{1} \mapsto e_{11}+e_{33}, e_{2} \mapsto \frac{\sqrt{2}}{2}\left(e_{12}-e_{23}\right), \\
e_{3} \mapsto \frac{\sqrt{2}}{2}\left(e_{12}+e_{23}\right), e_{4} \mapsto e_{13}\end{array}$ & \\
\hline $\begin{array}{l}\mathfrak{g}_{8}(\alpha) \\
\left(\alpha \neq \frac{1}{4}\right)\end{array}$ & 3 & $\begin{array}{c}e_{1} \mapsto x_{1} e_{11}-x_{2} e_{33}, \\
e_{2} \mapsto(1-4 \alpha)^{-\frac{1}{4}}\left(e_{12}+e_{23}\right), \\
e_{3} \mapsto(1-4 \alpha)^{-\frac{1}{4}} x_{1} e_{12} \\
+(1-4 \alpha)^{-\frac{1}{4}} x_{2} e_{23}, e_{4} \mapsto e_{14}, \text { where } \\
x_{1}=\frac{1}{2}\left(1-(1-4 \alpha)^{\frac{1}{2}}\right), \\
x_{2}=\frac{1}{2}\left(1+(1-4 \alpha)^{\frac{1}{2}}\right) \\
\end{array}$ & \\
\hline $\mathfrak{g}_{8}\left(\frac{1}{4}\right)$ & 4 & $\begin{array}{c}e_{1} \mapsto \frac{1}{2}\left(e_{11}-e_{44}\right)+e_{23} \\
e_{2} \mapsto e_{12}+e_{13}+e_{24}+e_{34} \\
e_{3} \mapsto \frac{1}{2}\left(e_{12}-e_{13}+3 e_{24}+e_{34}\right) \\
e_{4} \mapsto 2 e_{14}\end{array}$ & $\begin{array}{c}e_{1} * e_{1}=-\frac{1}{2} e_{1}-\frac{1}{4} e_{2}+\frac{1}{2} e_{3}+\frac{1}{2} e_{4}, \\
e_{1} * e_{2}=-\frac{1}{2} e_{2}+e_{3}+e_{4}, \\
e_{1} * e_{3}=-\frac{1}{4} e_{2}+\frac{1}{2} e_{3}+\frac{1}{4} e_{4}, \\
e_{1} * e_{4}=\frac{1}{2} e_{4}, e_{2} * e_{1}=-\frac{1}{2} e_{2}+e_{4} \\
e_{2} * e_{2}=e_{4}, e_{2} * e_{3}=e_{4} \\
e_{3} * e_{1}=-\frac{1}{2} e_{3}+\frac{1}{4} e_{4} \\
e_{3} * e_{3}=\frac{1}{4} e_{4}, e_{4} * e_{1}=-\frac{1}{2} e_{4}\end{array}$ \\
\hline
\end{tabular}

\section{4. ÉTALE AFFINE REPRESENTATIONS AND CORRESPONDING LEFT-SYMMETRIC} ALGEBRAS

Obviously, the construction of faithful representations at the end of last section is not unique (in fact, there are a lot of faithful representations). In this paper, we hope that the faithful representation in the case $\operatorname{dim} \mathfrak{g}=\mu(\mathfrak{g})$ can induce an étale affine representation. In general, the construction is not trivial (unfortunately at many cases we cannot use the construction given in some proofs in section 3). For example, if the adjoint representation of $\mathfrak{g}$ is faithful (hence $\mathfrak{g}$ is centerless), it cannot induce an étale affine representation. Otherwise, in this case, there exists an invertible derivation of $\mathfrak{g}$ (by equation $(2.5), q^{-1}$ is a derivation) which implies $\mathfrak{g}$ is nilpotent. Hence $\mathfrak{g}$ has a non-zero center, which is a contradiction. Furthermore, we would also like to point out that although the étale affine representations are not unique, either, our construction has a natural and simple form as follows (that is, the "construction rule"). 
Theorem 4.1. For the faithful representations given in section 3 (as in Table 3), if $\mu(\mathfrak{g})=\operatorname{dim} \mathfrak{g}$, then there exists an étale affine representation $\Phi=(\rho, q): \mathfrak{g} \rightarrow \mathfrak{g r}(V) \oplus V$ with base zero, where $\rho: \mathfrak{g} \rightarrow \mathfrak{g l}(V)$ is just the given faithful representation and

$$
q(x)=\rho(x)\left(\sum_{i=1}^{n} e_{i}\right) .
$$

Proof Obviously, $q$ satisfies the equation (2.5). $q$ is a linear isomorphism follows from the direct computation.

We also list the corresponding left-symmetric algebra structures (with non-zero products) in the fourth column in Table 3 by equation (2.6).

On the other hand, besides the semisimple Lie algebra $\mathfrak{s l}(2, \mathbb{C})$ (there does not exist a compatible left-symmetric algebra structure $[\mathrm{M}]$ ), for the Lie algebras with $\operatorname{dim} \mathfrak{g}>$ $\mu(\mathfrak{g})$ in Table 3, we can also construct (in some sense, like a kind of "lifting") a faithful representation $(\rho, V)$ with the same dimension of $\mathfrak{g}$ and it can induce an étale affine representation $\Phi$ which still satisfies the demands in Theorem 4.1. That is, $\Phi=(\rho, q)$ : $\mathfrak{g} \rightarrow \mathfrak{g l}(V) \oplus V$, where $q(x)=\rho(x)\left(\sum_{i=1}^{n} e_{i}\right)$.

In Table 4, for those Lie algebras, we list $\operatorname{dim} \mathfrak{g}$ in column 2 , faithful representation $\rho: \mathfrak{g} \rightarrow \mathfrak{g l}(\operatorname{dim} \mathfrak{g}, \mathbb{C})$ in column 3 and the corresponding left-symmetric algebra (with non-zero products) in column 4 .

TABLE 4

\begin{tabular}{|c|c|c|c|}
\hline$\overline{\mathfrak{g}}$ & $\operatorname{dim} \mathfrak{g}$ & Faithful representation & Left-symmetric algebra \\
\hline $\mathfrak{r}_{2}(\mathbb{C}) \oplus \mathbb{C}$ & 3 & $e_{1} \mapsto e_{12}, e_{2} \mapsto e_{22}, e_{3} \mapsto e_{33}$ & $\begin{array}{c}e_{1} * e_{2}=e_{1}, e_{2} * e_{2}=e_{2} \\
e_{3} * e_{3}=e_{3}\end{array}$ \\
\hline $\mathfrak{n}_{3}(\mathbb{C}) \oplus \mathbb{C}$ & 4 & $\begin{array}{c}e_{1} \mapsto e_{11}+e_{22}+e_{33}+e_{12}-e_{23}, \\
e_{2} \mapsto e_{12}+e_{23}, e_{3} \mapsto 2 e_{13}, \\
e_{4} \mapsto e_{44}\end{array}$ & $\begin{array}{c}e_{1} * e_{1}=e_{1}-e_{2}+\frac{1}{2} e_{3}, \\
e_{1} * e_{2}=e_{2}+\frac{1}{2} e_{3}, e_{1} * e_{3}=e_{3}, \\
e_{2} * e_{1}=e_{2}-\frac{1}{2} e_{3}, e_{2} * e_{2}=\frac{1}{2} e_{3}, \\
e_{3} * e_{1}=e_{3}, e_{4} * e_{4}=e_{4}\end{array}$ \\
\hline $\mathfrak{r}_{2}(\mathbb{C}) \oplus \mathbb{C}^{2}$ & 4 & $\begin{aligned} e_{1} \mapsto e_{12}, e_{2} \mapsto e_{22}, \\
e_{3} \mapsto e_{33}, e_{4} \mapsto e_{44}\end{aligned}$ & $\begin{array}{l}e_{1} * e_{2}=e_{1}, e_{2} * e_{2}=e_{2}, \\
e_{3} * e_{3}=e_{3}, e_{4} * e_{4}=e_{4}\end{array}$ \\
\hline $\mathfrak{r}_{3}(\mathbb{C}) \oplus \mathbb{C}$ & 4 & $\begin{array}{c}e_{1} \mapsto e_{12}-e_{33}, e_{2} \mapsto e_{13}, \\
e_{3} \mapsto e_{23}, e_{4} \mapsto e_{44}\end{array}$ & $\begin{array}{c}e_{1} * e_{1}=-e_{1}+e_{2}, e_{1} * e_{3}=e_{2}, \\
e_{2} * e_{1}=-e_{2}, e_{3} * e_{1}=-e_{3}, \\
e_{4} * e_{4}=e_{4}\end{array}$ \\
\hline $\mathfrak{r}_{3, \lambda}(\mathbb{C}) \oplus \mathbb{C}$ & 4 & $\begin{array}{c}e_{1} \mapsto-e_{11}+(\lambda-1) e_{33} \\
e_{2} \mapsto e_{21}, e_{3} \mapsto e_{31}, e_{4} \mapsto e_{44}\end{array}$ & $\begin{array}{c}e_{1} * e_{1}=-e_{1}+\left(\lambda^{2}-\lambda\right) e_{3} \\
e_{1} * e_{3}=(\lambda-1) e_{3}, e_{2} * e_{1}=-e_{2} \\
e_{3} * e_{1}=-e_{3}, e_{4} * e_{4}=e_{4}\end{array}$ \\
\hline $\mathfrak{r}_{2}(\mathbb{C}) \oplus \mathfrak{r}_{2}(\mathbb{C})$ & 4 & $\begin{array}{l}e_{1} \mapsto e_{12}, e_{2} \mapsto e_{22}, \\
e_{3} \mapsto e_{34}, e_{4} \mapsto e_{44}\end{array}$ & $\begin{array}{l}e_{1} * e_{2}=e_{1}, e_{2} * e_{2}=e_{2} \\
e_{3} * e_{4}=e_{3}, e_{4} * e_{4}=e_{4}\end{array}$ \\
\hline $\mathfrak{s l}_{2}(\mathbb{C}) \oplus \mathbb{C}$ & 4 & $\begin{array}{c}e_{1} \mapsto 2 e_{12}+\frac{1}{2} e_{34}, \\
e_{2} \mapsto \frac{1}{2} e_{21}+2 e_{43}, \\
e_{3} \mapsto e_{11}-e_{22}+e_{33}-e_{44}, \\
e_{4} \mapsto I_{4}\end{array}$ & $\begin{array}{c}e_{1} * e_{2}=\frac{1}{2}\left(e_{3}+e_{4}\right), e_{1} * e_{3}=-e_{1}, \\
e_{1} * e_{4}=e_{1}, e_{2} * e_{1}=\frac{1}{2}\left(-e_{3}+e_{4}\right), \\
e_{2} * e_{3}=e_{2}, e_{2} * e_{4}=e_{2}, \\
e_{3} * e_{1}=e_{1}, e_{3} * e_{2}=-e_{2}, \\
e_{3} * e_{3}=e_{4}, e_{3} * e_{4}=e_{3}, \\
e_{4} * e_{1}=e_{1}, e_{4} * e_{2}=e_{2}, \\
e_{4} * e_{3}=e_{3}, e_{4} * e_{4}=e_{4}\end{array}$ \\
\hline $\mathfrak{g}_{6}$ & 4 & $\begin{aligned} e_{1} & \mapsto e_{11}-e_{44}, \\
e_{2} & \mapsto 2 e_{12}+e_{34}, \\
e_{3} & \mapsto e_{13}+e_{24}, \\
& e_{4} \mapsto e_{14}\end{aligned}$ & $\begin{array}{c}e_{1} * e_{1}=2 e_{4}-e_{1}, e_{1} * e_{2}=2 e_{4}, \\
e_{1} * e_{3}=e_{4}, e_{1} * e_{4}=e_{4} \\
e_{2} * e_{1}=2 e_{4}-e_{2}, e_{2} * e_{3}=2 e_{4}, \\
e_{3} * e_{1}=e_{4}-e_{3}, e_{3} * e_{2}=e_{4} \\
e_{4} * e_{1}=-e_{4}\end{array}$ \\
\hline
\end{tabular}


TABLE 4 (Continued)

\begin{tabular}{|c|c|c|c|}
\hline $\mathfrak{g}$ & $\operatorname{dim} \mathfrak{g}$ & Faithful representation & Left-symmetric algebra \\
\hline $\mathfrak{g}_{7}$ & 4 & $e_{1} \mapsto e_{11}+2 e_{33}+e_{44}$, & $e_{1} * e_{1}=e_{1}+e_{2}+e_{3}-e_{4}$, \\
& & $e_{2} \mapsto e_{12}-e_{24}+e_{34}$, & $e_{1} * e_{2}=e_{2}+e_{3}-\frac{1}{2} e_{4}$, \\
& & $e_{3} \mapsto e_{12}+e_{24}+e_{34}$, & $e_{1} * e_{3}=e_{2}+e_{3}-\frac{1}{2} e_{4}$, \\
& & $e_{4} \mapsto 2 e_{14}$ & $e_{1} * e_{4}=e_{4}, e_{2} * e_{1}=e_{2}-\frac{1}{2} e_{4}$, \\
& & $e_{2} * e_{2}=-\frac{1}{2} e_{4}, e_{2} * e_{3}=\frac{1}{2} e_{4}$, \\
& & & $e_{3} * e_{1}=e_{3}-\frac{1}{2} e_{4}, e_{3} * e_{2}=-\frac{1}{2} e_{4}$, \\
& & & $e_{3} * e_{3}=\frac{1}{2} e_{4}, e_{4} * e_{1}=e_{4}$ \\
\hline $\mathfrak{g}_{8}(\alpha)$ & 4 & $e_{1} \mapsto(x-1) e_{22}-x e_{33}-e_{44}$, & $e_{1} * e_{1}=-e_{1}-\alpha e_{2}+\frac{x}{2 x-1} e_{4}$, \\
$\left(\alpha \neq \frac{1}{4}\right)$ & & $e_{2} \mapsto e_{12}+e_{24}+e_{34}$, & $e_{1} * e_{2}=-e_{2}+e_{3}+\frac{x}{2 x-1} e_{4}$, \\
& & $e_{3} \mapsto(1-x) e_{12}+x_{24}$ & $e_{1} * e_{3}=-\alpha e_{2}+\frac{\alpha}{2 x-1} e_{4}$, \\
& & $(1-x) e_{34}, e_{4} \mapsto(2 x-1) e_{14}$, & $e_{2} * e_{1}=-e_{2}+\frac{x}{2 x-1} e_{4}$, \\
& & where $x=\frac{1}{2}(1+\sqrt{(1-4 \alpha)})$ & $e_{2} * e_{2}=\frac{1}{2 x-1} e_{4}, e_{2} * e_{3}=\frac{x}{2 x-1} e_{4}$, \\
& & $e_{3} * e_{1}=-e_{3}+\frac{\alpha}{2 x-1} e_{4}$, \\
& & & $e_{3} * e_{2}=\frac{1-x}{2 x-1} e_{4}, e_{3} * e_{3}=\frac{\alpha}{2 x-1} e_{4}$, \\
& & & $e_{4} * e_{1}=-e_{4}$ \\
\hline
\end{tabular}

\section{ACKNOWLEDGEMENTS}

We thank Professor D. Burde for introducing us the history of refining Ado's Theorem, bringing our attention to the reference [GST] and the valuable discussion. We also thank the referee for the important suggestion. This work was supported in part by the National Natural Science Foundation of China (10501025, 10571091, 10621101), NKBRPC (2006CB805905), Program for New Century Excellent Talents in University, Liu Hui Center for Applied Mathematics and Youth Teachers Foundation of Tianjin University.

\section{REFERENCES}

[Bai] C.M. Bai, Left-symmetric algebras from linear functions, J. Algebra 281 (2004)651-665.

[BM1] C.M. Bai, D.J. Meng, The structure of bi-symmetric algebras and their sub-adjacent Lie algebras, Comm. in Algebra 28 (2000) 2717-2734.

[BM2] C.M. Bai, D.J. Meng, Left-symmetric algebras and complete Lie algebras, Comm. in Algebra 30 (2002) 1001-1015.

[BM3] C.M. Bai, D.J. Meng, A Lie algebraic approach to Novikov algebras, J. Geo. Phys. 45 (2003) $218-230$.

[BN] A.A. Balinskii, S.P. Novikov, Poisson brackets of hydrodynamic type, Frobenius algebras and Lie algebras, Soviet Math. Dokl. 32 (1985) 228-231.

[Bo] M. Bordemann, Generalized Lax pairs, the modified classical Yang-Baxter equation, and affine geometry of Lie groups, Comm. Math. Phys. 135 (1990) 201-216.

[Bu1] D. Burde, Left-invariant affine structures on nilpotent Lie groups, Habilitationsschrift, Dusseldorf, 1998.

[Bu2] D. Burde, A refinement of Ado's Theorem, Archiv Math. 70 (1998)119-127.

[Bu3] D. Burde, Affine structures on nilmanifolds, Int. J. Math. 7 (1996) 599-616.

[Bu4] D. Burde, Simple left-symmetric algebras with solvable Lie algebra, Manuscipta Math. 95 (1998) 397-411.

[BS] D. Burde, C. Steinhoff, Classification of orbit closures of 4-dimensional complex Lie algebras, J. Algebra 214 (1999) 729-739.

[CL] F. Chapoton, M. Livernet, Pre-Lie algebras and the rooted trees operad, Int. Math. Res. Not. (2001) 395-408.

[CK] A. Connes, D. Kreimer, Hopf algebras, renormalization and noncommutative geometry, Comm. Math. Phys. 199 (1998) 203-242. 
[DM1] J.M. Dardie, A. Medina, Double extension symplectique d'un groupe de Lie symplectique, Adv. Math. 117 (1996) 208-227.

[DM2] J.M. Dardie, A. Medina, Algebres de Lie kähleriennes et double extension, J. Algebra 185 (1995) 774-795.

[ES] P. Etingof, A. Soloviev, Quantization of geometric classical r-matrix, Math. Res. Lett. 6 (1999) 223-228.

[GST] R. Ghanam, I. Strugar, G. Thompson, Matrix representations for low-dimensional Lie algebras, Extracta Mathematicae Vol. 20 No. 2 (2005) 151-184.

[GS] I.Z. Golubschik, V.V. Sokolov, Generalized operator Yang-Baxter equations, integrable ODES and nonassociative algebras, J. Nonlinear Math. Phys., 7 (2000) 184-197.

[J] N. Jacobson, Lie algebras, Interscience, New York (1962).

$[\mathrm{K}] \quad$ H. Kim, Complete left-invariant affine structures on nilpotent Lie groups, J. Differential Geometry 24 (1986) 373-394.

[M] A. Medina, Flat left-invariant connections adapted to the automorphism structure of a Lie group, J. Differential Geometry 16 (1981) 445-474.

[R] B.E. Reed, Representations of solvable Lie algebras, Michigan Math. J. 16 (1969) 227-233.

[V] E.B. Vinberg, Convex homogeneous cones, Transl. of Moscow Math. Soc. No. 12 (1963) 340-403.

[Z] E.I. Zel'manov, On a class of local translation invariant Lie algebras, Soviet Math. Dokl. 35 (1987) 216-218.

Department of Mathematics, Tianjin University, Tianjin, 300072, PR China

E-mail address: kangyf@tju.edu.cn

Chern Institute of Mathematics \& LPMC, Nankai University, Tianjin, 300071, PR China

E-mail address: baicm@nankai.edu.cn 